\title{
Time-frequency mapping of the positional characteristics of single- and multimode wave beam at the lengthy atmospheric path output
}

\author{
Tatiana Arsenyan ${ }^{2}$, Eugeniy Babanin ${ }^{2, \star}$, Vitaliy Kapranov ${ }^{1}$, Rinat Stryungis ${ }^{2}$, \\ Natalia Suhareva ${ }^{2}$, and Vjatcheslav Tugaenko ${ }^{1}$ \\ ${ }^{1}$ S.P. Korolev Rocket and Space Corporation "Energia" (Russian Federation) \\ ${ }^{2}$ M.V. Lomonosov Moscow State University (Russian Federation)
}

\begin{abstract}
The comparative experimental analysis of the single-mode and multimode positional characteristics of the wave beams at the lengthy atmospheric path output was carried out and the results are presented. As the main instrument the method of nonlinear time-frequency mapping was chosen using Wigner function apparatus. The characteristic time-frequency spectra under the conditions of strong, intermediate and weak turbulence of the atmospheric path were defined.
\end{abstract}

The broad range of challenges suggested by the optical propagation channel demands quick signal correction to compensate the atmosphere induced distortions. In this article the sequential model for the analysis of the positional characteristics of the wave beams using the nonlinear time-frequency mapping is presented.

Let us construct the time-space maps for nonstationary processes of the variations of a wave beam spatial moments on the base of Wigner-Willy representation. This allows circumventing restrictions of the uncertainty principle for time and frequency resolution [1]:

$$
W V T_{s}(t, \omega)=\int_{-\infty}^{\infty} s(t+\tau / 2) \cdot s^{*}(t-\tau / 2) e^{-i \omega \tau} d \tau
$$

Here $s(t)$ is the initial signal. The presence of strong interference distortions makes the experimental results more difficult to interpret.

Traditionally for meteomonitoring they use the $C_{n}^{2}$ parameter or the Rytov parameter $\beta^{2}$. There is a three interval classification in radio physical applications that determines the Rytov parameter values corresponding to weak - $[0,0.3)$, average - $[0.3,1)$ and strong - $[1, \ldots)$ turbulence development $[2]$

The time-frequency spectrum also depends on the atmospheric conditions. The first special moments spectra describe "the translational" beam motion. The spectra of the second moments describe the vibrational modes of the collimated beam and its nondiagonal element $x y$ Var describes "rotatory" movement thus changing the direction of the orientation axis of approximating ellipsoid.

Under the conditions described the sampling rate was equal to $100 \mathrm{~Hz}$. In the case of weak turbulence the main components of the spectrum appear to be in the frequency range up to $5 \mathrm{~Hz}$ (Fig.1)). When the Rytov parameter increases up to 2.5 the spectrum occupies all the frequency range observed.

^e-mail: babanin.eugen@gmail.com 

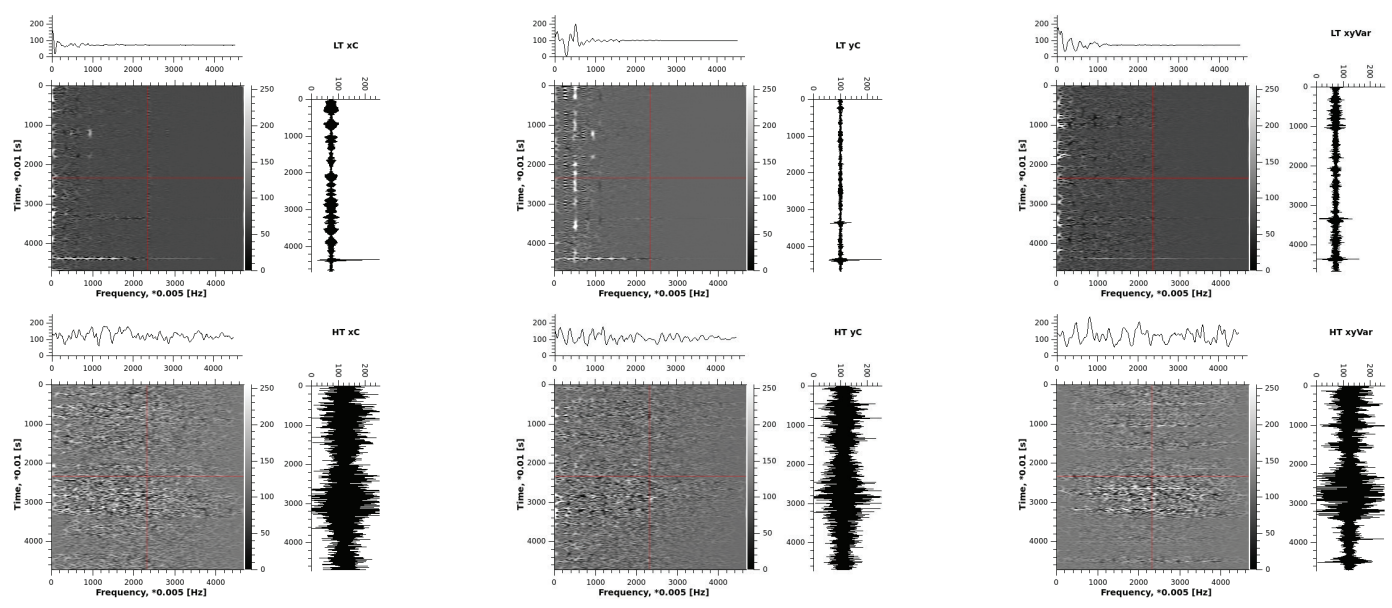

Figure 1. Time-frequency spectra for components of first moment and nondiagonal element of second moment in conditions of low (top three figures) and hight turbulence (bottom three figyres).

A multimode wave beam was used for meteomonitoring tasks and its diameter increased the registration plane size [2]. The time-frequency spectra of the illumination variance presented on Fig. 2 were obtained in conditions corresponding to three turbulence levels. The sampling rate was equal to $500 \mathrm{~Hz}$. When the Rytov parameter increases the spectrum occupies the higher frequency range. Moreover for multimode beam the time-frequency spectrum depends on the square frame liner size. When we increase the frame size the spectrum shifts to the low frequency area.
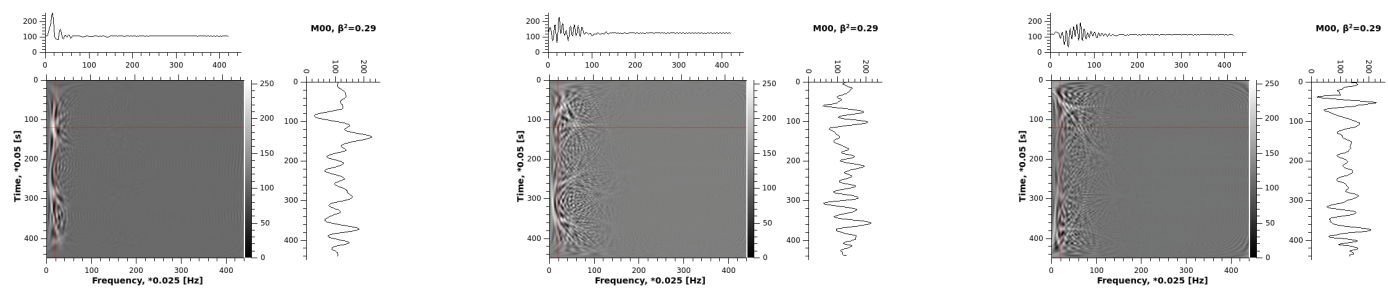

Figure 2. Time-frequency spectra for components of zero moment in conditions of low, average and hight turbulence for the $16 \times 16 \mathrm{~mm}$ square aperture.

The work was supported by Russian Foundation for Basic Research. RFBR Project A-15-0807484 .

\section{References}

[1] Cohen L.,Time Frequency Analysis: Theory and Applications, Prentice Hall, 1994.

[2] E. A. Babanin, V. V. Kapranov, N. A. Suhareva, V. Y. Tugaenko, O. M. Vokhnik "Space-temporal stochastic characteristics of complex amplitude for the sounding vector optical beam", Proc. of PIERS 2017. 